\title{
Design of ethylene oxide production process based on adaptive design of experiments and Bayesian optimization
}

\author{
Ryo Iwama ${ }^{1}$ and Hiromasa Kaneko ${ }^{1}$ \\ ${ }^{1}$ Meiji University
}

September 24, 2020

\begin{abstract}
In process design, the values of design variables $\mathrm{X}$ for equipment and operating conditions should be optimized for entire processes, including all unit operations, such as reactors and distillation columns, to consider effects between unit operations. However, as the number of $\mathrm{X}$ increases, many more simulations are required to search for the optimal $\mathrm{X}$ values. Furthermore, multiple objective variables $\mathrm{Y}$, such as yields, make the optimization problem difficult. We propose a process design method based on adaptive design of experiments and Bayesian optimization. Optimization of $\mathrm{X}$ values that satisfy target values of multiple $\mathrm{Y}$ variables are searched, and simulations for the optimized $\mathrm{X}$ values are then repeated. Therefore, $\mathrm{X}$ will be optimized by a small number of simulations. We verify the effectiveness of this method by simulating an ethylene oxide production plant.
\end{abstract}

\section{Introduction}

Process design involves the entire procedure from raw materials to products, combining multiple unit operations, such as reaction, heat exchange, and separation. In the development of new production processes, the design and performance of virtual plants are simulated. In this study, the objective is to design an efficient ethylene oxide (EO) production process.

EO is an important petrochemical product formed by the oxidation of ethylene. It is converted to various compounds such as ethylene glycol and ethanolamine, and is a starting material for fibers and detergents. EO production is about $9,000 \mathrm{t} / \mathrm{y}^{1}$ in Japan. In the production process, the oxidation of ethylene is accompanied by side reactions:

$2 \mathrm{C}_{2} \mathrm{H}_{4}+\mathrm{O}_{2} \rightarrow 2 \mathrm{C}_{2} \mathrm{H}_{4} \mathrm{O}(1)$

$\mathrm{C}_{2} \mathrm{H}_{4}+3 \mathrm{O}_{2} \rightarrow 2 \mathrm{CO}_{2}+2 \mathrm{H}_{2} \mathrm{O}(2)$

$2 \mathrm{C}_{2} \mathrm{H}_{4} \mathrm{O}+5 \mathrm{O}_{2} \rightarrow 4 \mathrm{CO}_{2}+4 \mathrm{H}_{2} \mathrm{O}(3)$

Eq. (1) is the main reaction to produce EO and it requires silver catalysts $\left(\mathrm{Ag}_{2} \mathrm{O}\right)$. Eqs. $(2,3)$ are side reactions that produce carbon dioxide and water. The EO process has a risk of explosion because Eq. (2) has a heat reaction of $+1323 \mathrm{~kJ} / \mathrm{mol}$ at $523 \mathrm{~K}$. Therefore, there is a restriction on reactor inlet compositions and conversions ${ }^{2}$. As a result, unreacted gas is recycled, and the reactor exhibits nonlinear and complex behaviors. Here, we design the EO production process assuming that only these three reactions proceed.

Much research has been conducted to improve the performance of EO production. Zhou et al. used a reactor model to optimize design variables such as inlet compositions of ethylene, oxygen, and carbon dioxide, as well as reactor temperature and the amount of ethylene dichloride ${ }^{3}$. Lahiri et al. optimized design variables, such as inlet compositions and gas flow, using a support vector machine and a genetic algorithm for EO 
recycling. They verified the results with an actual plant based on the optimized design variables, and reported improved EO production rate and catalyst selectivity ${ }^{4}$. Yang et al. performed modeling and validations for steady-state, dynamic, and start-up operation conditions with EO recycling. They speeded up the plant start-up with optimized start-up profiles for inlet compositions, temperature, and pressure ${ }^{5}$. Rahimpour et al. modeled catalyst deactivation using artificial neural networks to determine the optimal amount of ethylene dichloride ${ }^{6}$. Luo et al. integrated reaction kinetics, a reactor model, and a catalyst deactivation model of an industrial ethylene oxide reactor and compared the results with an actual EO plant ${ }^{7}$. Nawaz et al. used Aspen Custom Modeler to optimize several design variables, such as reactor temperature and inlet compositions $^{8}$. Peschel et al. considered interactions between reaction concepts and the entire EO process, and modeled the physical and chemical principles. They reported reduced operating costs and reduced carbon dioxide emissions ${ }^{9}$. Distillation columns for the removal of carbon dioxide were optimized for several variables, such as temperature and pressure ${ }^{10,11}$.

Often, the values of design variables are optimized for each unit, such as a reactor and a distillation column traditionally. Hence, effects between units are not considered, and the optimized values of design variables from these methods are not always accurate when considering the entire process.

We propose a method for optimizing the entire process by considering effects between the units. Therefore, all units must be optimized simultaneously, with a very large number of design variables that need to be optimized. For example, the design variables X for the EO process, such as equipment and operating conditions, have 23 parameters. Assuming there are ten candidates for each X, the total number of combinations would be $10^{23}$, which requires a huge number of simulations.

Hence, we propose a process design method based on Bayesian optimization ${ }^{12}$ where $\mathrm{X}$ values that satisfy target values of multiple objective variables $\mathrm{Y}$, such as yield, are searched. Then $\mathrm{X}$ will be optimized with a small number of simulations. We verify the effectiveness of the proposed method by simulating an EO plant.

\section{Methods}

First, the target EO process is described. Then, after the design of experiments (DoE) and the adaptive design of experiments are described, the procedure for combining these methods is discussed.

Ethylene Oxide (EO) process

The process flow diagram (PFD) of the designed EO process is shown in Figure 1. Here, the products are 99.5 wt- $\%$ EO and a 10.0 mol-\% aqueous EO solution ${ }^{13}$. In the EO reactions, it is assumed that a silver catalyst is used. Ethylene and oxygen are supplied to a plug-flow reactor (R1) and catalyst at a high temperature and pressure, and are converted into EO. The produced EO is then absorbed by water in the absorption column (T1). As noted above, there is a risk of explosion in the reactor because of the large number of exothermic reactions; hence, there are restrictions on conversions and reactor inlet compositions. The unreacted gases are recycled and purged with raw materials, such as argon or ethane, and the carbon dioxide by-product is removed before they are supplied to the reactor again. Ethylene oxide absorbed in water is removed from the water (T2), and from light hydrocarbons (T3), and rectified in the distillation column (T4). 


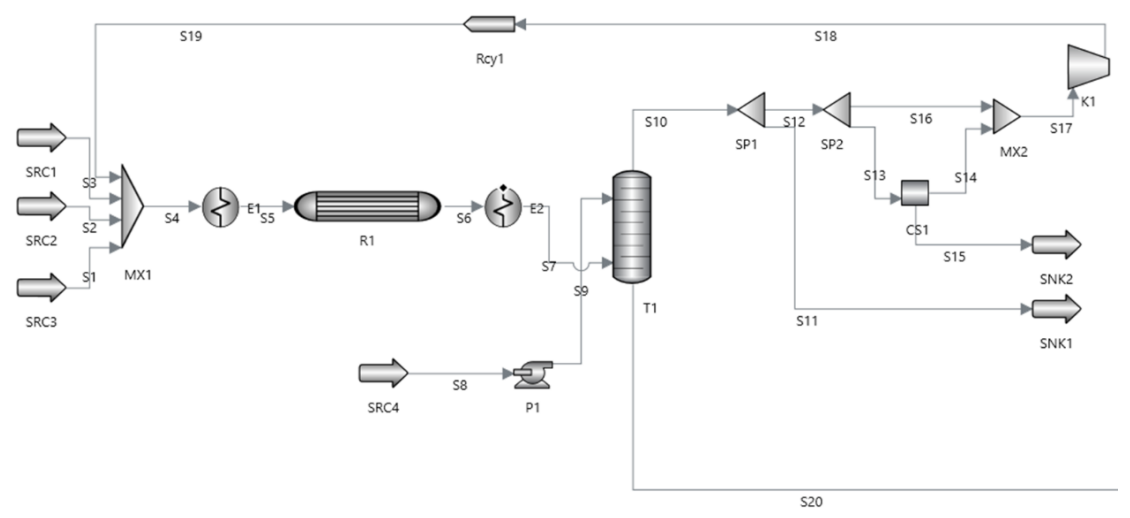

(a) Reaction section

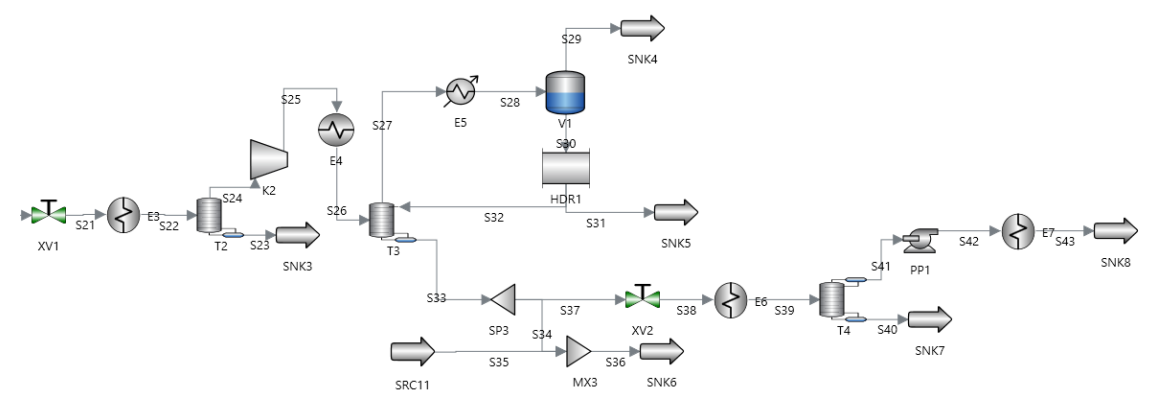

(b) Separation section.

Figure 1. Process flow diagram of ethylene oxide production.

Design of experiments (DoE)

To reduce the number of simulations or experiments, a DoE was developed. It is a method for determining which candidates are to be experimented first among experimental candidates. Here, we adopt the D-optimal $\operatorname{design}^{13}$ in the DoE, which can select several informative $\mathrm{X}$ values from a large number of candidates. Therefore, it is possible to select the $\mathrm{X}$ candidates that are likely to construct a good regression model with only the dataset of X.

Adaptive DoE

An adaptive DoE that decides on the experimental candidates sequentially, on the basis of the experimental results, was developed. Response surface methodology ${ }^{15}$ and Bayesian optimization ${ }^{12}$ are often used in adaptive DoE. Here, we use Bayesian optimization that can estimate regions with a small number of samples or extrapolation regions and can estimate from a small number of samples. Bayesian optimization has been used to search for optimal experimental conditions ${ }^{16,17,18,19}$ and process conditions ${ }^{20,21,22}$, and to optimize hyperparameters ${ }^{23}$. Here, we apply Bayesian optimization to process design.

Gaussian process regression (GPR)

Bayesian optimization uses Gaussian process regression $(\mathrm{GPR})^{24}$. When the input $\mathbf{x}$ is given, the output $\mathrm{y}(\mathbf{x}$ ) is represented as a probability model that follows a normal distribution. Assuming that the GPR model is linear, the i-th samples are given by:

$y^{(i)}=\mathbf{x}^{(i)} \mathbf{b}(4)$ 
where $\mathbf{b}$ is a vector of regression coefficients. The prior distribution of $\mathbf{b}$ assumes a normal distribution with a zero mean and variance $\sigma_{\mathrm{b}}{ }^{2}$. Then, the mean vector $m_{i}$ of the ${ }^{(i)}$ and the covariance $\sigma_{\mathrm{y} i, j}{ }^{2}$ of the $y^{(i)}$ and $y^{(j)}$ are calculated from:

$m_{i}=E\left[y^{(i)}\right]=0(4)$

$\sigma_{y i, j}{ }^{2}=\operatorname{cov}\left[y^{(i)}, y^{(j)}\right]=\mathbf{x}^{(i)} \mathbf{x}^{(j)^{T}} \sigma_{b}^{2}(5)$

The input $\mathbf{x}$ is transformed by the nonlinear function $\varphi$, and $\sigma_{\mathrm{y} i, j}{ }^{2}$ is calculated from:

$\sigma_{y i, j}{ }^{2}=\varphi\left(\mathbf{x}^{(i)}\right) \varphi\left(\mathbf{x}^{(j)}\right)^{T} \sigma_{b}^{2}(6)$

Actual y have a measurement error. The i-th samples including the measurement error is $y$ obs ${ }^{(i)}$ and its measurement error is $e^{(i)}$. Thus, $y$ obs $^{(i)}$ is given by:

$y_{\text {obs }}^{(i)}=y^{(i)}+e^{(i)}(7)$

$e^{(i)}$ assumes a normal distribution with a zero mean and variance $\sigma_{\mathrm{e}}{ }^{2}$, and $e^{(i)}$ is independent for each sample. Then, the covariance $\sigma$ yobs, $i, j{ }^{2}$ between $y$ obs $^{(i)}$ and $y$ obs $^{(j)}$ is calculated as follows:

$\sigma_{\text {yobs } i, j}{ }^{2}=\varphi\left(\mathbf{x}^{(i)}\right) \varphi\left(\mathbf{x}^{(j)}\right)^{T} \sigma_{b}^{2}+\delta_{i, j} \sigma_{e}^{2}=K\left(\mathbf{x}^{(i)}, \mathbf{x}^{(j)}\right)(8)$

where, $K$ is a kernel function.

In the GPR method, if the outputy $\mathbf{o b s}=\left(y_{\mathrm{obs}}{ }^{(1)} \cdots y_{\mathrm{obs}}{ }^{(n)}\right)^{T}$ corresponding to the past input vector $\mathbf{x}^{(1)} \cdots \mathbf{x}^{(n)}$ is used as training data, the output for the new input vector $\mathbf{x}^{(n+1)}$ can be estimated as a normal distribution with mean $m\left(\mathbf{x}^{(n+1)}\right)$ and variance $\sigma^{2}\left(\mathbf{x}^{(n+1)}\right)$, and is estimated by:

$m\left(\mathbf{x}^{(n+1)}\right)=\mathbf{k} \sum_{n}^{-1} \mathbf{y}_{\text {obs }}(9)$

$\sigma^{2}\left(\mathbf{x}^{(n+1)}\right)=K\left(\mathbf{x}^{(n+1)}, \mathbf{x}^{(n+1)}\right)-\mathbf{k} \sum_{n}^{-1} \mathbf{k}^{T}(10)$

subject to:

$\mathbf{k}=\left[K\left(\mathbf{x}^{(1)}, \mathbf{x}^{(n+1)}\right) \cdots K\left(\mathbf{x}^{(i)}, \mathbf{x}^{(n+1)}\right) \cdots K\left(\mathbf{x}^{(n)}, \mathbf{x}^{(n+1)}\right)\right](11)$

Probability in target range

For the evaluation of each candidate in Bayesian optimization, the probability $P$ that each $\mathrm{y}$ is within the range $\left(y_{\min } \leq y \leq y_{\max }\right)$ is calculated ${ }^{25}$. It assumed that outputy ${ }^{(i)}$ is a normal distribution with mean $m\left(\mathbf{x}_{\text {new }}{ }^{(i)}\right)$ and variance $\sigma^{2}\left(\mathbf{x}_{\text {new }}{ }^{(i)}\right)$ when a new sample $\mathbf{x}$ new ${ }^{(i)}$ is input into the GPR model. $P$ is thus calculated by integrating the area of the normal distribution, as given by:

$P\left(\mathbf{x}_{\text {new }}{ }^{(i)}\right)=\int_{y_{-} \min }^{y_{\text {max }}} \frac{1}{\sqrt{2 \pi \sigma^{2}\left(\mathbf{x}_{\text {new }}{ }^{(i)}\right)}} \exp \left\{-\frac{1}{2 \sigma^{2}\left(\mathbf{x}_{\text {new }}{ }^{(i)}\right)}\left(y-m\left(\mathbf{x}_{\text {new }}{ }^{(i)}\right)\right)^{2}\right\} \mathrm{dy}(12)$

Figure 2 shows the basic concept of $P$. When the predicted values are close to the target range of y, the probability of candidates like $c_{1}$, which has $m(\mathbf{x})$ that are close to the target range of $\mathrm{y}$, is large; and when the predicted values are far from the target range of $\mathrm{y}$, the probability of candidates like $c_{2}$ with high $\sigma^{2}(\mathbf{x})$ is large. 


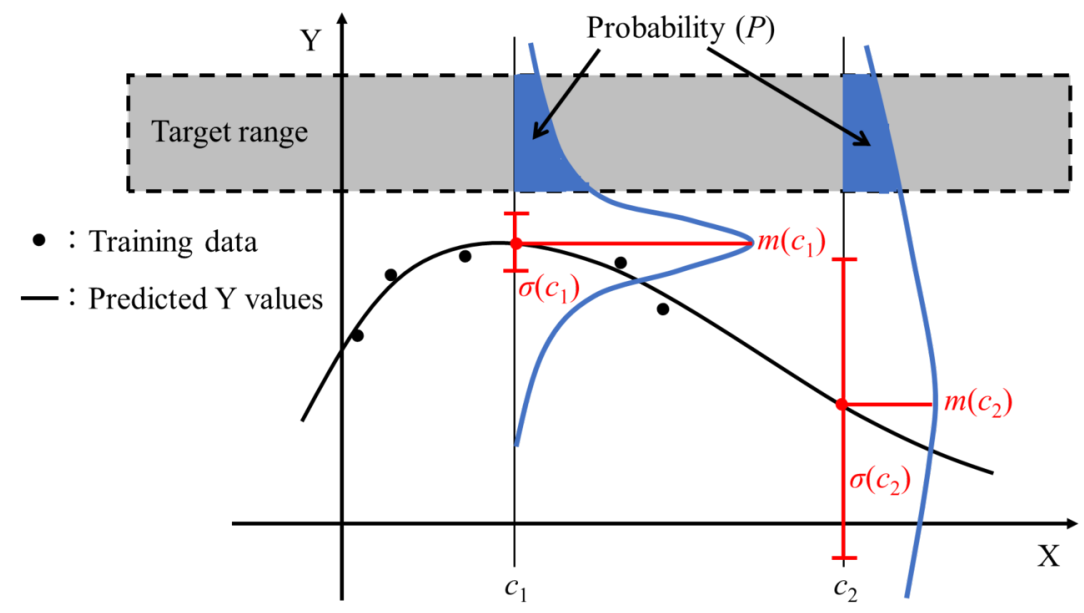

Figure 2. The basic concept of probability $P$. Black dots is known data, red dots are unknown data, the black line is predicted values by the GPR method, and the blue lines are normal distributions for unknown data.

\section{Proposed method}

We propose a process design method based on Bayesian optimization, as shown in the outline in Figure 3. First, the EO process is designed, and one million candidates of $\mathrm{X}$ are generated by random numbers. Then, 50 candidates of $\mathrm{X}$ are selected by using D-optimal design, and the EO plant is simulated with those candidates, yielding $\mathrm{Y}$ values. The GPR model is constructed between $\mathrm{X}$ and $\mathrm{Y}$ with 50 samples, and $P$ defined by Eq. (12) is calculated using output from the model. Because there are multiple Y variables, the acquisition function is the product of $P$ for all $\mathrm{Y}$ variables. However, multiplying values less than one will result in smaller values; therefore, the acquisition function is the sum of $\log (P)$ values. In addition, because the target ranges of $\mathrm{Y}$ are different, the acquisition function is scaled by using the probabilities in the target range of the GPR model samples $\left[P\left(\mathbf{x}_{\text {train }}\right)\right]$. This acquisition function $P$ all is calculated as follows:

$P_{\text {all }}\left(\mathbf{x}_{\text {new }}(i)\right)=\log \left(\frac{P(\mathbf{x})_{1}-\min \left(P\left(\mathbf{x}_{\text {train }}\right)_{1}\right)}{\max \left(P\left(\mathbf{x}_{\text {train }}\right)_{1}\right)-\min \left(P\left(\mathbf{x}_{\text {train }}\right)_{1}\right)}\right)+\cdots+\log \left(\frac{P(\mathbf{x})_{k}-\min \left(P\left(\mathbf{x}_{\text {train }}\right)_{k}\right)}{\max \left(P\left(\mathbf{x}_{\text {train }}\right)_{k}\right)-\min \left(P\left(\mathbf{x}_{\text {train }}\right)_{k}\right)}\right)$

where $k$ is the number of $\mathrm{Y}$. The EO plant is simulated with candidates of $\mathrm{X}$ having the highest values of $P$ all, and $\mathrm{Y}$ values are obtained. If the $\mathrm{Y}$ values do not achieve the targets, the GPR model is updated using simulation results, and this flow is repeated until all $\mathrm{Y}$ values are achieved within the targets. 


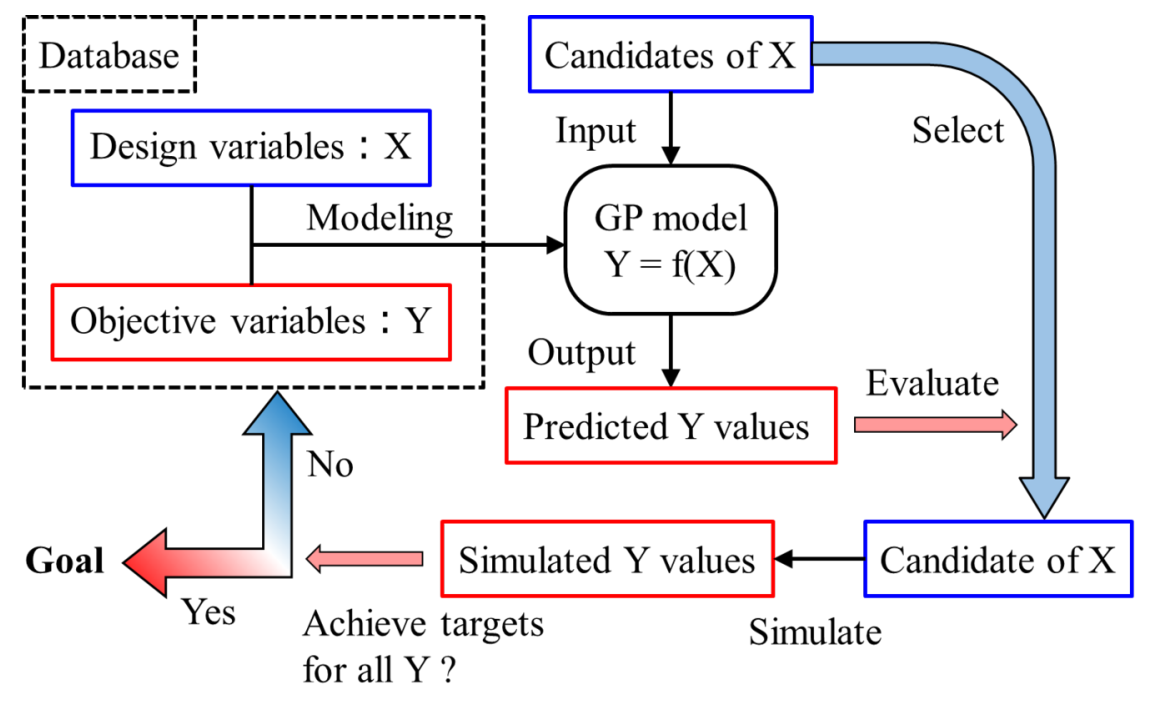

Figure 3. Outline of the proposed method

\section{Results and Discussion}

Table 1 shows the design variables $\mathrm{X}$ and their ranges. The $\mathrm{X}$ were defined as 24 variables based on equipment and operating conditions, such as the flow rate, reactor specifications, and distillation column specifications. Table 2 show $\mathrm{Y}$ and their target ranges. The $\mathrm{Y}$ were defined as eight variables, such as reactor inlet composition, conversion ratio, and product limitations. The reactor inlet concentration and conversion ratio were set because of explosion limits.

Table 1. The design variables $\mathrm{X}$ and their ranges

\begin{tabular}{llllll}
\hline $\mathrm{X}$ & Location & The design variables & Unit & Min & Max \\
\hline $\mathrm{X}_{1}$ & SRC1 & The feed of ethylene & $\mathrm{kmol} / \mathrm{h}$ & 500 & 800 \\
$\mathrm{X}_{2}$ & SRC2 & The feed of oxygen & $\mathrm{kmol} / \mathrm{h}$ & 550 & 850 \\
$\mathrm{X}_{3}$ & SRC3 & The feed of methane & $\mathrm{kmol} / \mathrm{h}$ & 250 & 450 \\
$\mathrm{X}_{4}$ & $\mathrm{R} 1$ & The number of tubes & - & 500 & 900 \\
$\mathrm{X}_{5}$ & $\mathrm{R} 1$ & Reactor length & $\mathrm{m}$ & 20.0 & 60.0 \\
$\mathrm{X}_{6}$ & $\mathrm{R} 1$ & Reactor temperature & $\mathrm{K}$ & 498 & 523 \\
$\mathrm{X}_{7}$ & $\mathrm{SRC} 4$ & The feed of water & $\mathrm{kmol} / \mathrm{h}$ & $1.00 \times 10^{3}$ & $2.00 \times 10^{3}$ \\
$\mathrm{X}_{8}$ & $\mathrm{~T} 1$ & The number of stages & - & 13 & 15 \\
$\mathrm{X}_{9}$ & $\mathrm{SP} 1$ & The rate of separation & - & 0.00100 & 0.0500 \\
$\mathrm{X}_{10}$ & $\mathrm{SP} 2$ & The rate of separation & - & 0.00100 & 0.500 \\
$\mathrm{X}_{11}$ & $\mathrm{~T} 2$ & Pressure & $\mathrm{bar}$ & 0.100 & 0.900 \\
$\mathrm{X}_{12}$ & $\mathrm{~T} 2$ & The number of stages & - & 13 & 15 \\
$\mathrm{X}_{13}$ & $\mathrm{~T} 2$ & The number of feed stages & - & 10 & 13 \\
$\mathrm{X}_{14}$ & $\mathrm{~T} 2$ & Bottom reflux ratio & - & 0.0500 & 1.50 \\
$\mathrm{X}_{15}$ & $\mathrm{~T} 3$ & Pressure & $\mathrm{bar}$ & 3.00 & 7.00 \\
$\mathrm{X}_{16}$ & $\mathrm{~T} 3$ & The number of stages & - & 13 & 15 \\
$\mathrm{X}_{17}$ & $\mathrm{~T} 3$ & The number of feed stages & - & 10 & 13 \\
$\mathrm{X}_{18}$ & $\mathrm{~T} 3$ & Bottom reflux ratio & - & 0.500 & 1.50 \\
$\mathrm{X}_{19}$ & $\mathrm{~T} 3$ & Vapor fraction & - & 0.100 & 0.700
\end{tabular}




\begin{tabular}{llllll}
\hline $\mathrm{X}$ & Location & The design variables & Unit & Min & Max \\
\hline $\mathrm{X}_{20}$ & $\mathrm{~T} 4$ & Pressure & bar & 0.100 & 0.900 \\
$\mathrm{X}_{21}$ & $\mathrm{~T} 4$ & The number of stages & - & 13 & 15 \\
$\mathrm{X}_{22}$ & $\mathrm{~T} 4$ & The number of feed stages & - & 10 & 13 \\
$\mathrm{X}_{23}$ & $\mathrm{~T} 4$ & Bottom reflux ratio & - & 0.100 & 1.00 \\
$\mathrm{X}_{24}$ & $\mathrm{~T} 4$ & Top reflux ratio & - & 0.500 & 2.00 \\
\hline
\end{tabular}

Table 2. The objective variables $\mathbf{Y}$ and their target ranges

\begin{tabular}{llllll}
\hline $\mathrm{Y}$ & Location & The design variables & Unit & Min & Max \\
\hline $\mathrm{Y}_{1}$ & $\mathrm{~S} 5$ & Reactor inlet concentration of ethylene & - & 0.260 & 0.280 \\
$\mathrm{Y}_{2}$ & $\mathrm{~S} 5$ & Reactor inlet concentration of oxygen & - & 0.0700 & 0.0800 \\
$\mathrm{Y}_{3}$ & $\mathrm{~S} 5$ & Reactor inlet concentration of $\mathrm{CO}_{2}$ & - & 0.0300 & 0.0600 \\
$\mathrm{Y}_{4}$ & $\mathrm{R} 1$ & Conversion ratio of ethylene & - & 0.0500 & 0.100 \\
$\mathrm{Y}_{5}$ & $\mathrm{R} 1$ & Conversion ratio of oxygen & - & 0.100 & 0.500 \\
$\mathrm{Y}_{6}$ & $\mathrm{~S} 36$ & The product 2 flow of EO & $\mathrm{kmol} / \mathrm{h}$ & 20.0 & 40.0 \\
$\mathrm{Y}_{7}$ & $\mathrm{~S} 43$ & The product 1 flow of EO & $\mathrm{kg} / \mathrm{h}$ & $4.38 \times 10^{3}$ & $5.00 \times 10^{3}$ \\
$\mathrm{Y}_{8}$ & $\mathrm{~S} 43$ & The product 1 concentration of EO & - & 0.995 & 1.00 \\
\hline
\end{tabular}

We used SimCentral ${ }^{26}$ simulation software. Robustness is important when the results are obtained by inputting random data into the process simulator. With SimCentral, we receive an error flag and can discard the results when the solution is divergent and cannot be obtained. In an iterative process, a sequential simulator takes a long time to converge; but a convergent solution can be obtained in a short time with SimCcntral. In addition, $\mathrm{X}$ can be set as needed in ADoE because the specifications can be freely changed. Furthermore, SimCentral can be performed using JavaScript and can be automated by association with Python programs, which means that the proposed method can be easily combined with SimCentral.

We succeeded in searching for $\mathrm{X}$ that achieved target ranges of $\mathrm{Y}$ using 139 simulations (D-optimal design required 50, and Bayesian optimization required 89). As a comparison method, $\mathrm{X}$ candidates were selected in a random search. In this case, the target ranges of the $\mathrm{Y}$ could not be achieved at all. Table 3 shows the result of 139 simulations, and Figure 4 shows the behavior of $Y$ for 139 simulations. In Bayesian optimization, the number of samples within the target range of $\mathrm{Y}$ increased with the number of trials. The accuracy of the GP model improved as the number of samples increased with the number of trials. The $\mathrm{Y}_{8}$ result, which has a narrow range, has a low probability and is not considered by $P$ all. Therefore, it was possible to treat each variable equally by performing range scaling, as shown in Eq (13).

Table 3. The results of $\mathrm{Y}$ that achieved targets

\begin{tabular}{lllll}
\hline $\mathrm{Y}$ & Unit & Min & Max & Result 1 \\
\hline $\mathrm{Y}_{1}-$ & 0.260 & 0.280 & 0.267 \\
$\mathrm{Y}_{2}$ & - & 0.0700 & 0.0800 & 0.0710 \\
$\mathrm{Y}_{3}-$ & 0.0300 & 0.0600 & 0.0395 \\
$\mathrm{Y}_{4}-$ & 0.0500 & 0.100 & 0.0682 \\
$\mathrm{Y}_{5}$ & - & 0.100 & 0.500 & 0.286 \\
$\mathrm{Y}_{6}$ & $\mathrm{kmol} / \mathrm{h}$ & 20.0 & 40.0 & 35.7 \\
$\mathrm{Y}_{7}$ & $\mathrm{~kg} / \mathrm{h}$ & $4.38 \times 10^{3}$ & $5.00 \times 10^{3}$ & $4.87 \times 10^{3}$ \\
$\mathrm{Y}_{8}$ & - & 0.995 & 1.00 & 0.996 \\
\hline
\end{tabular}



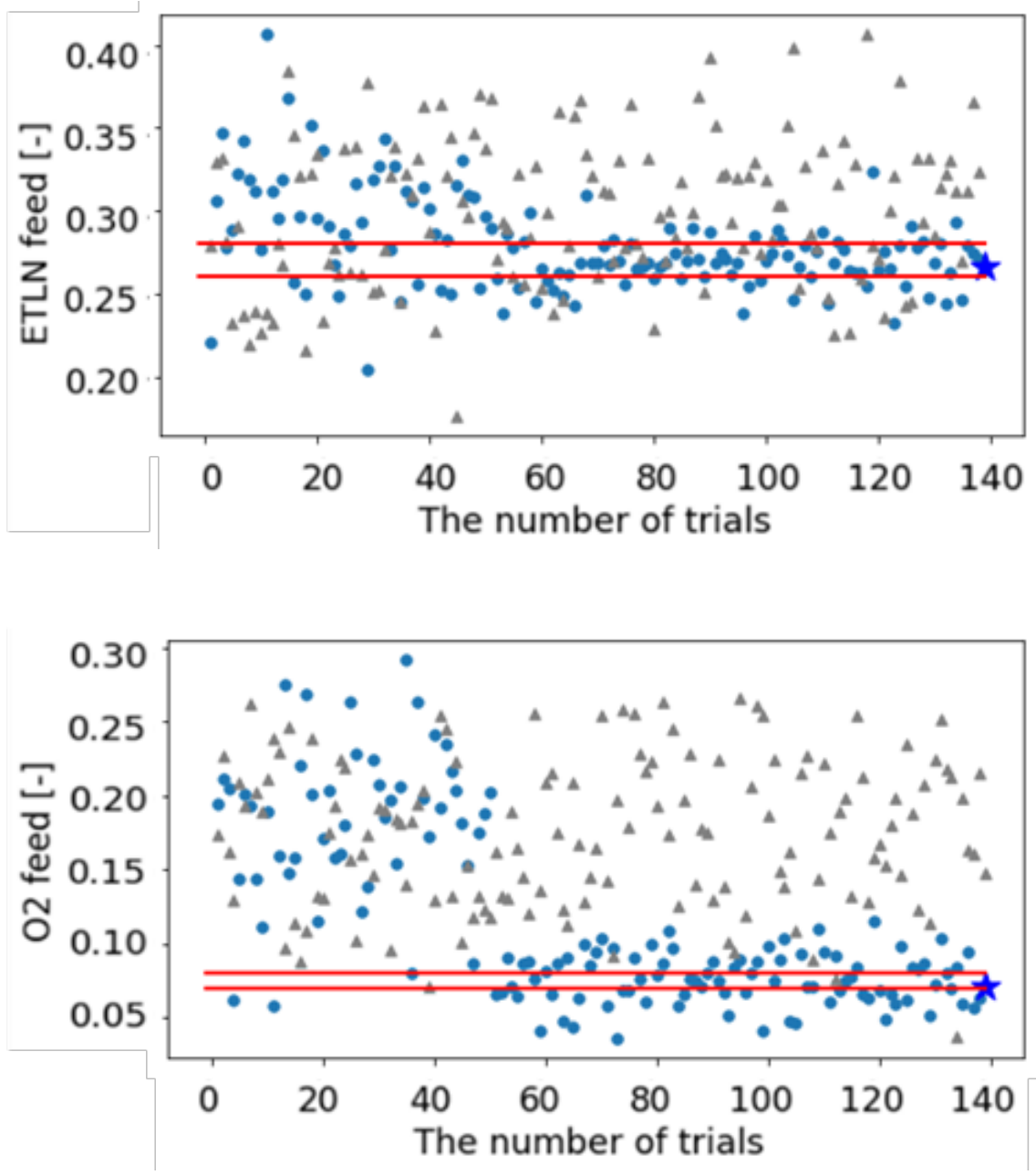

$\mathrm{Y}_{1}$ (b) $\mathrm{Y}_{2}$

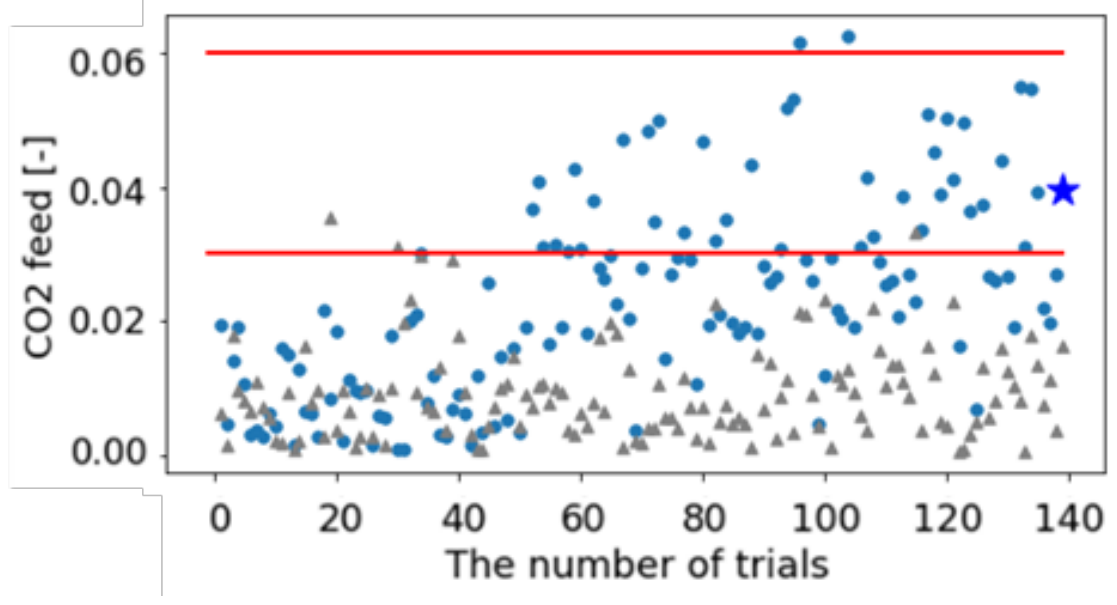




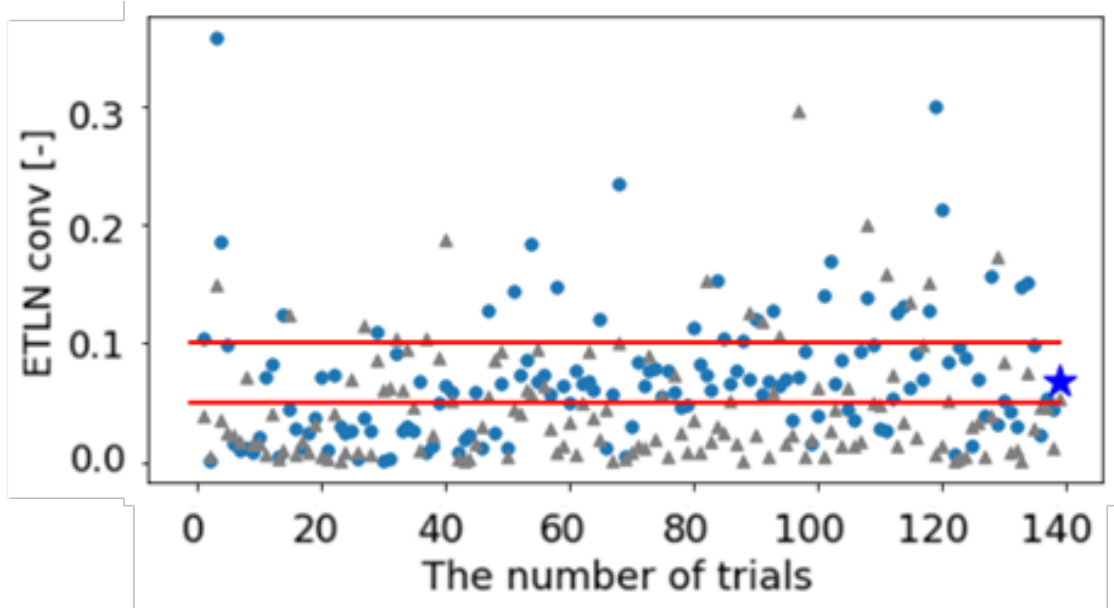

(c) $Y_{3}$ (d) $Y_{4}$
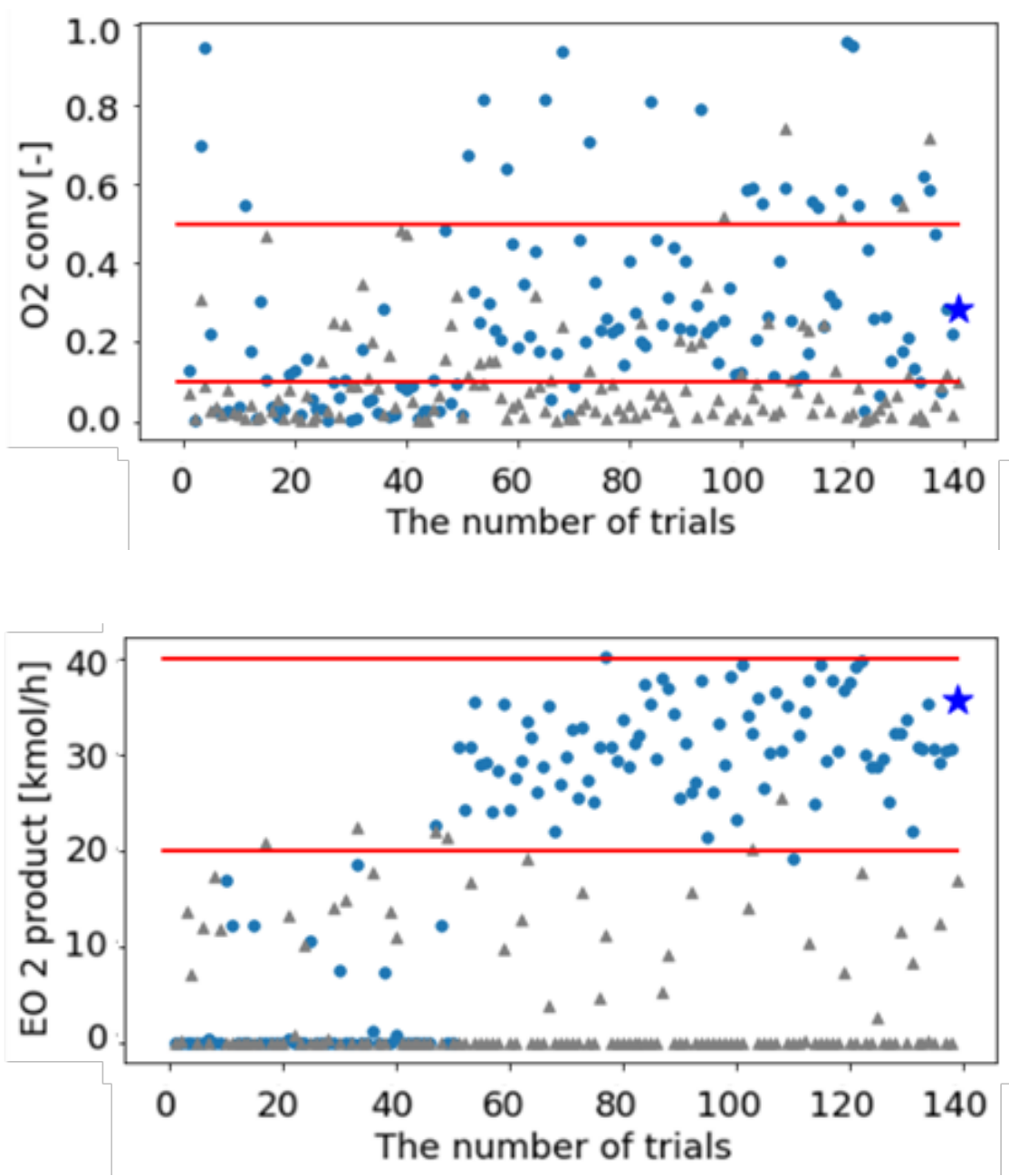

(e) $Y_{5}(f) Y_{6}$ 

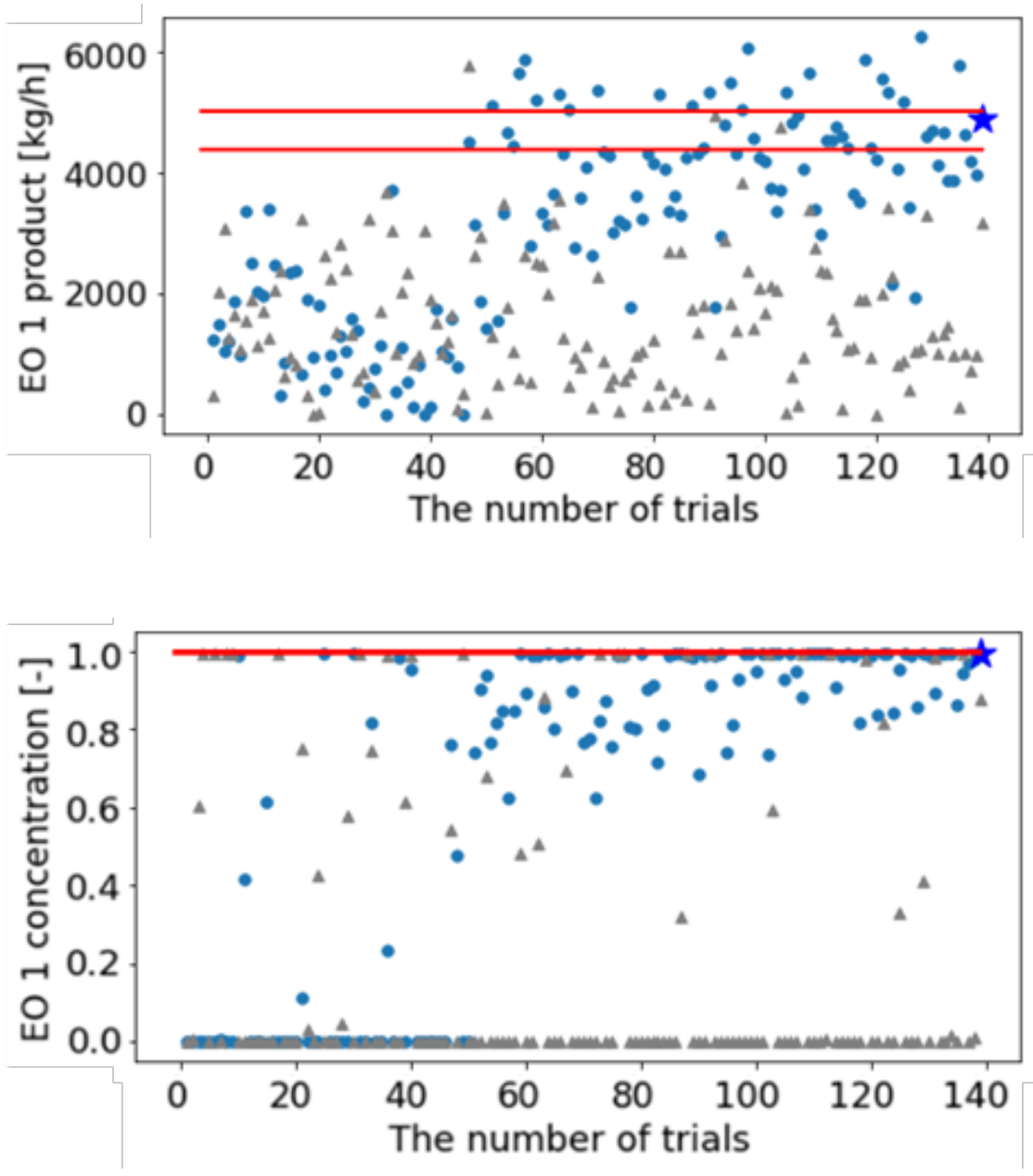

(g) $\mathrm{Y}_{7}(\mathrm{~h}) \mathrm{Y}_{8}$

Figure 4. Y values for 139 simulations. The blue circles indicate the Bayesian optimization results, the gray triangles indicate the random search results, the asterisks indicate the Bayesian optimization results that achieved the target ranges of $\mathrm{Y}$, and the red lines indicate the target ranges.

Bayesian optimization depends on the initial samples because it starts with a small number. Therefore, we changed the random value of D-optimal design, and performed Bayesian optimization again. The result is shown in Figure 5. In 83 simulations (D-optimal design took 50, and Bayesian optimization took 33), we succeeded in searching for $\mathrm{X}$ values that achieved target ranges of $\mathrm{Y}$. These $\mathrm{X}$ were the same as those before the random values were changed, indicating that the results of process design based on ADoE and Bayesian optimization were stable. A comparison of the results before and after changing the random values shows similar behavior, but the target was achieved faster after the change. The number of simulations decreased because there was a sample that could easily achieve the target in the initial samples. From the results shown in Figure 5, the reproducibility of the proposed method was confirmed.

We changed the candidates of $\mathrm{X}$ to one million samples and compared the results of the proposed method. Tables $(4,5)$ list the $\mathrm{Y}$ and $\mathrm{X}$ results, respectively. In the second set of results, 77 simulations (50 for D-optimal design, and 27 for Bayesian optimization) succeeded in searching for $\mathrm{X}$ that achieved the target ranges of Y. Similarly, we succeeded in the third set of results with 105 simulations (50 for D-optimal design, and 55 for Bayesian optimization). In Table 4, values of $\mathrm{Y}$ converged to relatively similar solutions. However, 
in Table 5, the values of $\mathrm{X}$ were dissimilar. In actuality, the final $\mathrm{X}$ values will be selected on the basis of cost comparisons or chemical engineering knowledge.
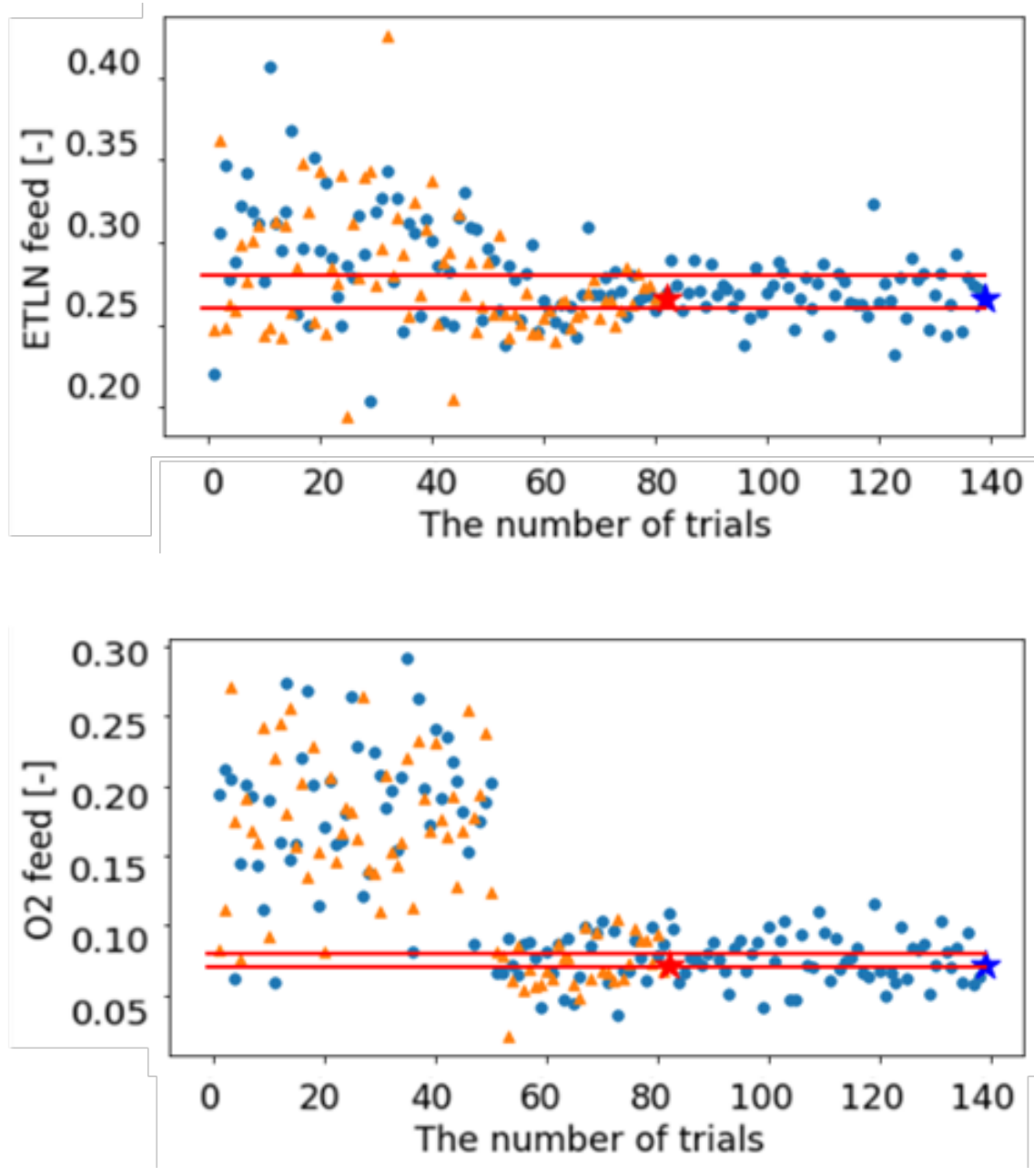

$\mathrm{Y}_{1}$ (b) $\mathrm{Y}_{2}$

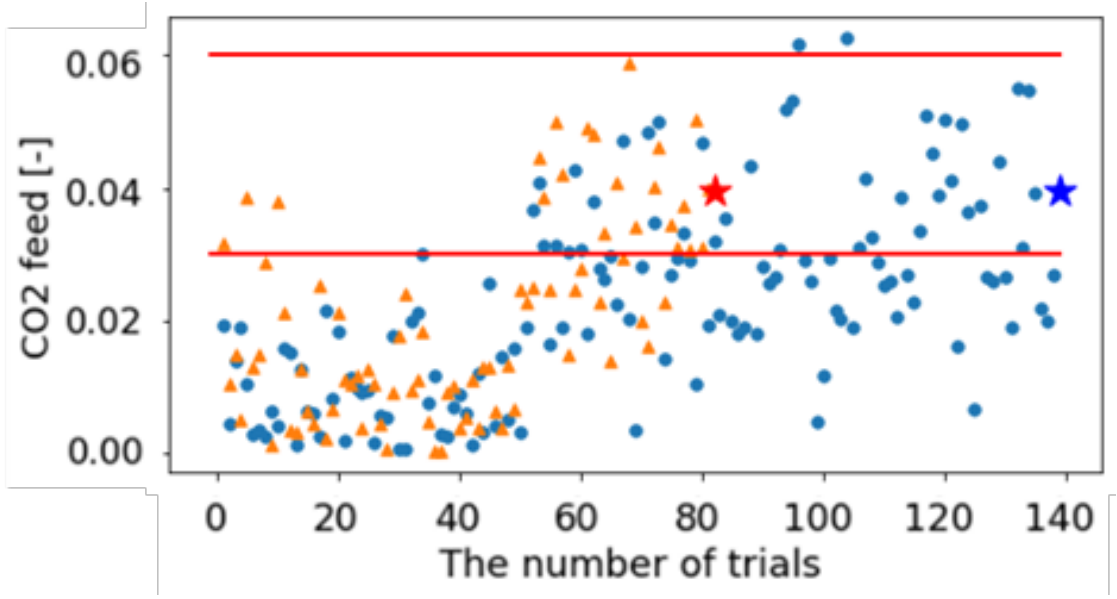




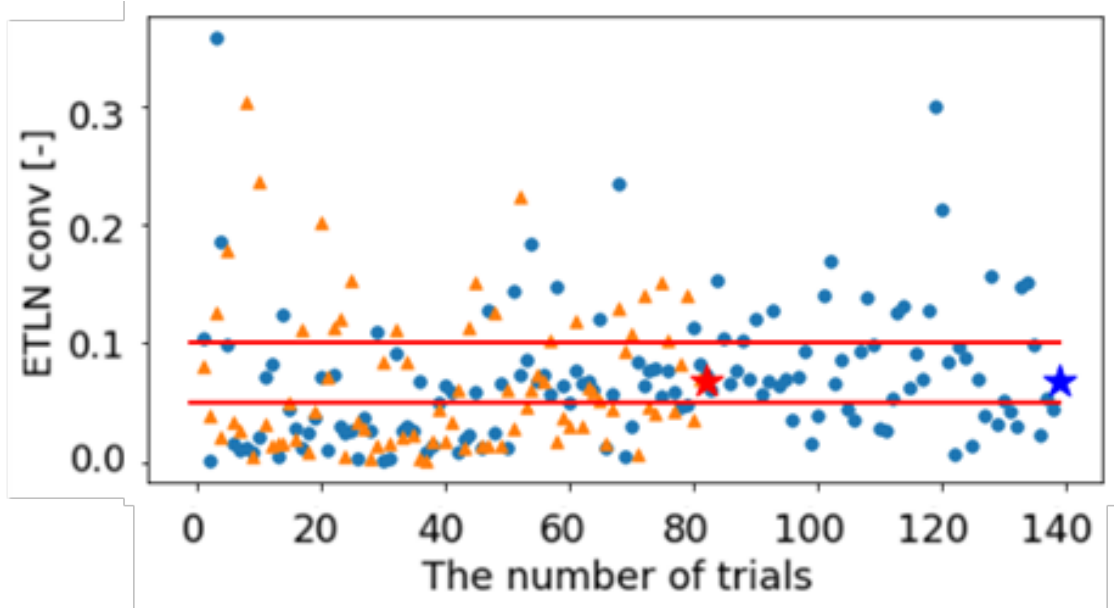

(c) $Y_{3}(d) Y_{4}$
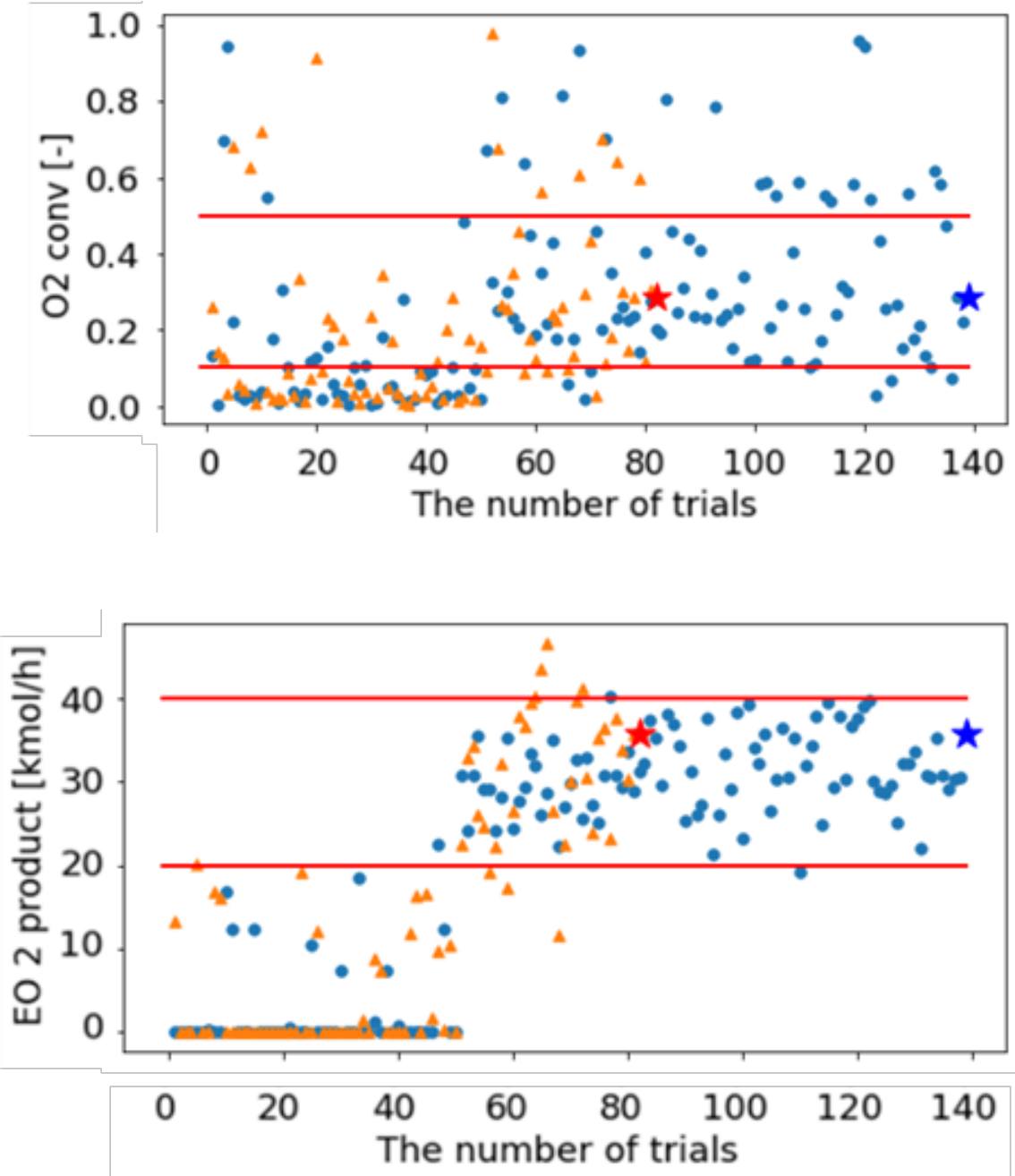
(e) $\mathrm{Y}_{5}(\mathrm{f}) \mathrm{Y}_{6}$
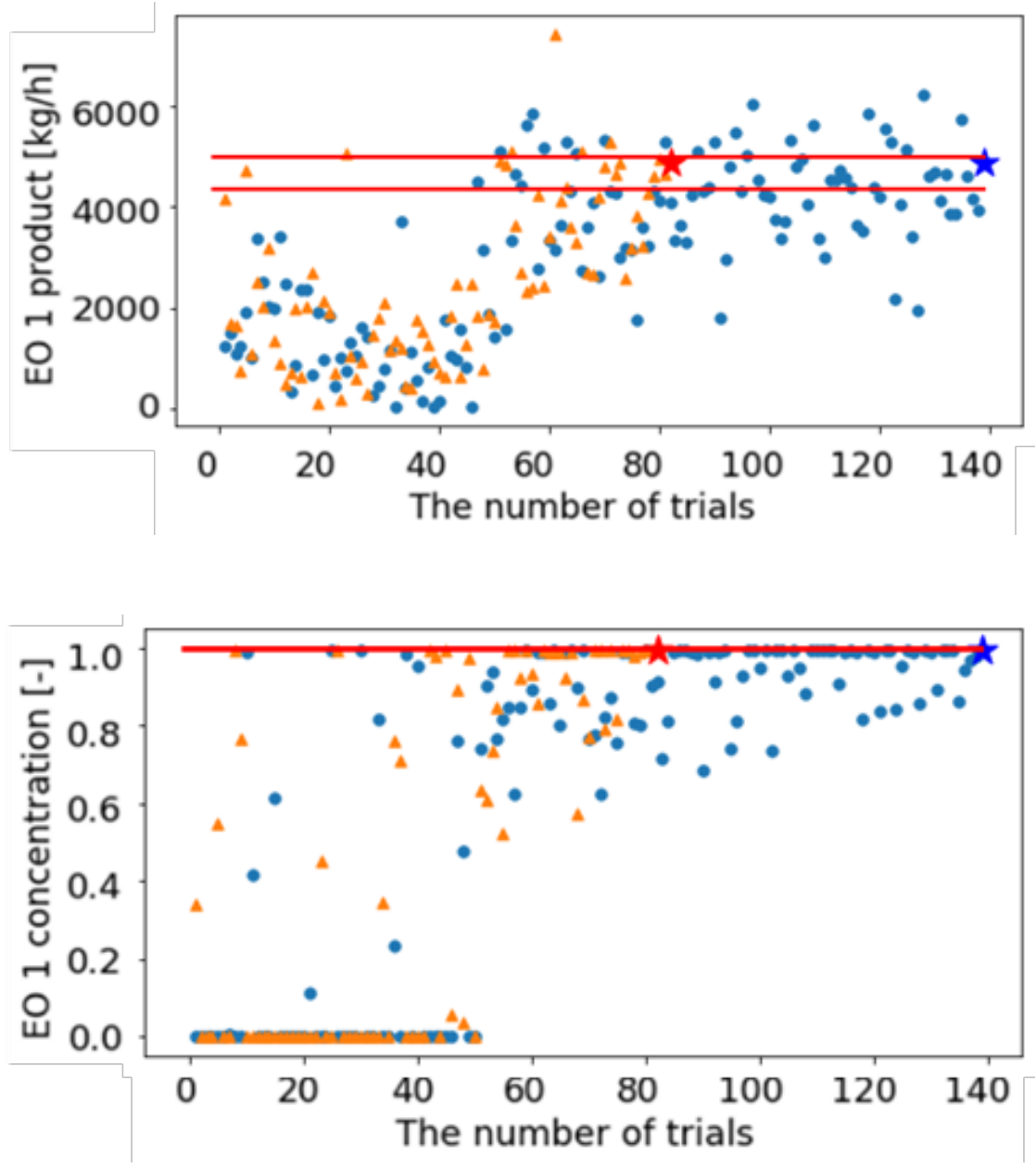

(g) $\mathrm{Y}_{7}(\mathrm{~h}) \mathrm{Y}_{8}$

Figure 5. Y values for 83 simulations. The blue circles indicate the Bayesian optimization results before changing the random values of D-optimal design, the orange triangles indicate the Bayesian optimization results after changing the random values of D-optimal design, the asterisks indicate the Bayesian optimization results that achieved the target ranges of $\mathrm{Y}$, and the red lines indicate the target ranges.

Table 4. Comparison of Y results

\begin{tabular}{lllllll}
\hline $\mathrm{Y}$ & Unit & Min & Max & Result_1 & Result_2 & Result_3 \\
\hline $\mathrm{Y}_{1}$ & - & 0.260 & 0.280 & 0.267 & 0.276 & 0.277 \\
$\mathrm{Y}_{2}$ & - & 0.0700 & 0.0800 & 0.0710 & 0.0708 & 0.0732 \\
$\mathrm{Y}_{3}$ & - & 0.0300 & 0.0600 & 0.0395 & 0.0430 & 0.0438 \\
$\mathrm{Y}_{4}$ & - & 0.0500 & 0.100 & 0.0682 & 0.0589 & 0.0641 \\
$\mathrm{Y}_{5}$ & - & 0.100 & 0.500 & 0.286 & 0.260 & 0.274 \\
$\mathrm{Y}_{6}$ & $\mathrm{kmol} / \mathrm{h}$ & 20.0 & 40.0 & 35.7 & 30.5 & 34.3 \\
$\mathrm{Y}_{7}$ & $\mathrm{~kg} / \mathrm{h}$ & $4.38 \times 10^{3}$ & $5.00 \times 10^{3}$ & $4.87 \times 10^{3}$ & $4.49 \times 10^{3}$ & $4.39 \times 10^{3}$ \\
$\mathrm{Y}_{8}$ & - & 0.995 & 1.00 & 0.996 & 0.995 & 0.995 \\
\hline
\end{tabular}


Table 5. Comparison of $\mathrm{X}$ results

\begin{tabular}{lllllll}
\hline $\mathrm{X}$ & Unit & Min & Max & Result_1 & Result_2 & Result_3 \\
\hline $\mathrm{X}_{1}$ & $\mathrm{kmol} / \mathrm{h}$ & 500 & 800 & 708 & 787 & 761 \\
$\mathrm{X}_{2}$ & $\mathrm{kmol} / \mathrm{h}$ & 550 & 850 & 727 & 736 & 808 \\
$\mathrm{X}_{3}$ & $\mathrm{kmol} / \mathrm{h}$ & 250 & 450 & 349 & 336 & 386 \\
$\mathrm{X}_{4}$ & - & 500 & 900 & 829 & 581 & 778 \\
$\mathrm{X}_{5}$ & $\mathrm{~m}$ & 20.0 & 60.0 & 47.8 & 58.0 & 53.4 \\
$\mathrm{X}_{6}$ & $\mathrm{~K}$ & 498 & 523 & 517 & 519 & 518.3 \\
$\mathrm{X}_{7}$ & $\mathrm{kmol} / \mathrm{h}$ & $1.00 \times 10^{3}$ & $2.00 \times 10^{3}$ & $1.20 \times 10^{3}$ & $1.01 \times 10^{3}$ & $1.21 \times 10^{3}$ \\
$\mathrm{X}_{8}$ & - & 13 & 15 & 13 & 13 & 14 \\
$\mathrm{X}_{9}$ & - & 0.00100 & 0.0500 & 0.0202 & 0.0423 & 0.0248 \\
$\mathrm{X}_{10}$ & - & 0.00100 & 0.500 & 0.0662 & 0.0360 & 0.0512 \\
$\mathrm{X}_{11}$ & bar & 0.100 & 0.900 & 0.400 & 0.270 & 0.241 \\
$\mathrm{X}_{12}$ & - & 13 & 15 & 13 & 13 & 14 \\
$\mathrm{X}_{13}$ & - & 10 & 13 & 10 & 12 & 11 \\
$\mathrm{X}_{14}$ & - & 0.0500 & 1.50 & 0.159 & 0.359 & 0.133 \\
$\mathrm{X}_{15}$ & bar & 3.00 & 7.00 & 5.42 & 3.97 & 4.306 \\
$\mathrm{X}_{16}$ & - & 13 & 15 & 13 & 13 & 13 \\
$\mathrm{X}_{17}$ & - & 10 & 13 & 11 & 12 & 11 \\
$\mathrm{X}_{18}$ & - & 0.500 & 1.50 & 1.03 & 0.760 & 1.35 \\
$\mathrm{X}_{19}$ & - & 0.100 & 0.700 & 0.131 & 0.108 & 0.132 \\
$\mathrm{X}_{20}$ & bar & 0.100 & 0.900 & 0.301 & 0.394 & 0.336 \\
$\mathrm{X}_{21}$ & - & 13 & 15 & 13 & 13 & 14 \\
$\mathrm{X}_{22}$ & - & 10 & 13 & 11 & 12 & 10 \\
$\mathrm{X}_{23}$ & - & 0.100 & 1.00 & 0.798 & 0.384 & 0.850 \\
$\mathrm{X}_{24}$ & - & 0.500 & 2.00 & 0.911 & 0.937 & 1.28 \\
\hline
\end{tabular}

\section{Conclusion}

We proposed a process design method that combines Bayesian optimization and a process simulator to search for design variables that satisfy the performances of an ethylene oxide plant with a small number of simulations. We verified the effectiveness of the method by comparing it with a random search. It was confirmed by the case study that the candidates for design variables that achieve the plant performances can be efficiently proposed. The reproducibility of the proposed method was also confirmed. Moreover, various candidates were obtained by increasing the number of Bayesian optimization trials. This method is expected to meet the needs of knowledgeable chemical engineers and to facilitate process designs of new plants.

\section{Acknowledgments}

This work was supported by JSPS KAKENHI Grant Number JP19K15352. We thank Alan Burns, PhD, from the Edanz Group (https://en-author-services.edanzgroup.com/ac) for editing a draft of this manuscript.

\section{References}

1. https://www.meti.go.jp/policy/mono_info_service/mono/chemistry/toukei_archives.html

2. Barecka MH, Skiborowski M, Gorak A, A novel approach for process retrofitting through process intensification: Ethylene oxide case study. Chem. Eng. Res. 2017;123:295-316 
3. Zhou XG, Yuan WK, Optimization of the fixed-bed reactor for ethylene epoxidation. Chem. Eng. Process. 2005;44(10):1098-1107

4. Lahiri SK, Khalfe N, PROCESS MODELING AND OPTIMIZATION OF INDUSTRIAL ETHYLENE OXIDE REACTOR BY INTEGRATING SUPPORT VECTOR REGRESSION AND GENETIC ALGORITHM. Can. J. Chem. Eng. 2009;87(1):118-128

5. Yang XT, Xu Q, Li KY, Sagar CD, Dynamic Simulation and Optimization for the Start-up Operation of An Ethylene Oxide Plant. Ind. Eng. Chem. Res. 2010;49(9):4360-4371

6. Rahimpour MR, Shayanmehr M, Nazari M, Modeling and Simulation of an Industrial Ethylene Oxide (EO) Reactor Using Artificial Neural Networks (ANN). Ind. Eng. Chem. Res. 2011;50(10):6044-6052

7. Luo N, Du WL, Ye ZC, Qian F, Development of a Hybrid Model for Industrial Ethylene Oxide Reactor. Ind. Eng. Chem. Res. 2012;51(19):6926-6932

8. Nawaz Z, Heterogeneous Reactor Modeling of an Industrial Multitubular Packed-Bed Ethylene Oxide Reactor. Chem. Eng. Technol. 2016;39(10):1845-1857

9. Peschel A, Jorke A, Sundmacher K, Freund H, Optimal reaction concept and plant wide optimization of the ethylene oxide process. Chem. Eng. J. 2012;207:656-674

10. Freguia S, Rochelle GT, Modeling of CO2 Capture by Aqueous Monoethanolamine. AIChE J. 2003;49(7):1676-1686

11. Jassim MS, Rochelle GT, Innovative absorber/stripper configurations for CO2 capture by aqueous monoethanolamine. Ind. Eng. Chem. Res. 2006;45(8):2465-2472

12. Shahriari B, Swersky K, Wang ZY, Adams RP, Freitas ND, Taking the Human Out of the Loop: A Review of Bayesian Optimization. Proceeding of the IEEE. 2016;104(1):148-175

13. http://scejcontest.chem-eng.kyushu-u.ac.jp/

14. Brandmaier S, Sahlin U, Tetko IV, Oberg T, PLS-Optimal: A Stepwise D-Optimal Design Based on Latent Variables. J. Chem. Inf. Model. 2012;52(4):975-983

15. Bezerra MA, Santelli RE, Oliveira EP, Villar LS, Escaleira LA, Response surface methodology (RSM) as a tool for optimization in analytical chemistry. Talanta. 2008;76(5):965-977

16. Niktin A, Fastovets I, Shadrin D, Pukalchik M, Oseledets I, Bayesian optimization for seed germination. Plants Methods. 2019;15:43

17. Tanaka R, Iwata H, Bayesian optimization for genomic selection: a method for discovering the best genotype among a large number of candidates. Theor. Appl. Genet. 2018;131(1):93-105

18. Pruksawan S, Lambard G, Samitsu S, Sodeyama K, Prediction and optimization of epoxy adhesive strength from a small dataset through active learning. STAM. 2019;20(1):1010-1021

19. Seko A, Togo A, Hayashi H, Tsuda K, Chaput L, Tanaka I, Prediction of Low-Thermal-Conductivity Compounds with First-Principles Anharmonic Lattice-Dynamics Calculations and Bayesian Optimization. Phys. Rev. Lett. 2015;115(20): 205901-1-5

20. Overstall AM, Woods DC, Martin KJ, Bayesian prediction for physical models with application to the optimization of the synthesis of pharmaceutical products using chemical kinetics. Comput. Statist. Data Anal. 2019;132:126-142

21. Chen HP, Bowels S, Zhang B, Fuhlbrigge T, Controller parameter optimization for complex industrial system with uncertainties. Measurement and control. 2019;52(7-8):888-895

22. Geng ZY, Yang F, Chen X, Wu NQ, Gaussian process based modeling and experimental design for sensor calibration in drifting environments. Sens. Actuators B. Chem. 2015;216:321-331

23. Katakami S, Sakamoto H, Okada M, Bayesian Hyperparameter Estimation using Gaussian Process and Bayesian Optimization. J. Phys. Soc. Jpn. 2019;88(7):2019

24. Rasmussen CE, Nickisch H, Gaussian Processes for Machine Learning (GPML) Toolbox. J. Mach. Learn. Res. 2010;11:3011-3015

25. Kishio T, Kaneko H, Funatsu K, Strategic parameter search method based on prediction errors and data density for efficient product design. Chemometr. Intell. Lab. Syst. 2013;127:70-79

26. AVEVA Process Simulation (formerly known as SimCentral)

\section{List of Figure Captions}


Figure 1. PFD of the EO process.

Figure 2. The basic concept of P. Black dots is known data, red dots are unknown data, black line is predicted values by GPR method, blue lines are normal distribution for unknown data.

Figure 3. The outline of the proposed method

Figure 4. Y values for 139 times simulation. The blue points indicate the Bayesian optimization results, the gray points indicate the random search results, the asterisks indicate the Bayesian optimization result achieved the target ranges of $\mathrm{Y}$, and the red lines indicate the target range.

Figure 5. Y values for 83 times simulation. The blue points indicate the Bayesian optimization results before changing the random value of $\mathrm{D}$-optimal design, the orange points indicate the Bayesian optimization results after changing the random value of D-optimal design, the asterisks indicate the Bayesian optimization result achieved the target ranges of $\mathrm{Y}$, and the red lines indicate the target range. 